\author{
S. Röser \\ Max-Planck-Institut für Astronomie \\ Heidelberg-Königstuhl, FRG
}

\title{
Introduction
}

In recent years satellite observations of the region of the Earth-Moon libration points $\mathrm{I}_{4}$ and $\mathrm{L}_{5}$ became available, which showed different results. Roach (1975) has measured perturbations of the Zodiacal light intensity near the libration points $\mathrm{L}_{4}$ and $\mathrm{L}_{5}$ with a photometer on board the OSO-6 satellite. He interpreted the results as counterglow of a cloud of particles, having an angular diameter of 6 degrees and an average brightness of $20 \mathrm{~S}_{10 \mathrm{~V}}$, whereas observations by Burnett et al. (1974) set an upper limit of $10 \mathrm{~S}_{10}$ blue. Earth bound observations made by Bruman (1969) with the 48-inch Palomar Schmidt telescope gave no indication for discrete objects nor for clouds.

Looking at the dynamical aspects of the matter, the circumstances are not favourable for a straightforward solution. In celestial mechanics there are great difficulties in treating a restricted $\left(\mathrm{m}_{4}=0\right)$ fourbody problem analytically, and though several papers are now present confirming the existence of periodic orbits around the libration points, even if eccentricity of the orbits of the primaries and the effect of radiation pressure are admitted (e.g. Matas, 1974), this does not confirm the existence of a cloud. Therefore we undertook a numerical approach to the problem, which enabled us to compare computed values for the mass supply of the cloud with measurements.

\section{Assumptions and calculations}

Besides the gravitational attraction of the Sun, the Earth and the Moon, we included in our calculations the effect of the Sun's radiation pressure using formulas given by Robertson (1937). For the equations of motiol we used cartesian relative coordinates originating in the Earth's centre. In order to get a good approximation to the real situation we made the following assumptions for the motion of the sun and the Moon. The Sun moves around the Earth on an elliptic orbit with eccentricity 0,0167 . 
The Moon's orbit has an inclination to the ecliptic of $5 .^{\circ} 1$, an eccentricity of 0.0549 , and the rotation of perigee and node are included. The initial positions of Sun, Earth and Moon were collinear, but this did not influence the results as has been shown by test calculations with different initial conditions.

The position of the libration point $\mathrm{L}_{4}$ was that of the elliptic restricted three-body problem, that means 60 degrees in advance of the Moon in the Moon's plane of orbit, and Earth, Moon and $\mathrm{L}_{4}$ form an equilateral triangle at any time. The probe began its motion at various positions at and near $\mathrm{L}_{4}$, the initial velocities being varied. Starting with these initial conditions at time $t=0$ the integration was performed in positive direction of time and was stopped, whenever the particle left the Earth-Moon system or hit one of the main bodies. Beginning at the same initial position and integrating backwards in time we found the origin of particles that could possibly contribute to a libration cloud.

For the numerical treatment of the problem a fourth order Runge-Kutta scheme with automatical step-size control has been applied. To insure the correctness of the integration procedure we reversed the direction of time at the final point of the integration and pursued the path back again until we reached $t=0$. We only used the results if no coordinate of the particle deviated from its original value more than 10 per cent.

\section{The lifetime of the particles}

The influence of radiation pressure on a particle depends on its size, mass-density and chemistry. For the calculations we adopted the parameter $1-\mu=\frac{\text { radiation pressure }}{\text { gravity }}$, which can be transformed into particle sizes via the rule of thumb

$$
\rho d=\frac{C}{1-\mu}
$$

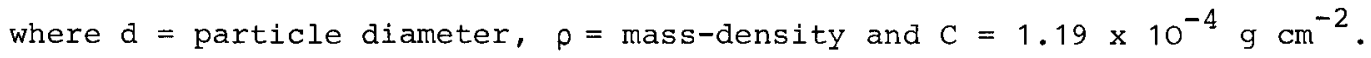
We made calculations for 8 values of $1-\mu$, the largest being 0.1 . Test computations showed that for larger values of this parameter the perturbations were too strong and the particles left the region around $\mathrm{L}_{4}$ immediately. From this we conclude that particles with a diameter smaller than about $3 \mu \mathrm{m}\left(\rho=3.5 \mathrm{~g} \mathrm{~cm}^{-3}\right)$ cannot contribute to a libration cloud. For each value of 1- $\mu \quad 59$ different initial conditions in position and velocity of the probe have been considered. 
Table 1 Life - times of the particles near the Earth - Moon libration point. T tot is the total lifetime of the particles, $T_{1}$ the time of residence in an area of $50 \times 26$ degrees ${ }^{2}$ around $L_{4}$ at distances between .8 and 1.2 (unit of length = mean distance Moon - Earth) and $\mathrm{T}_{2}$ the time of residence in a 1 degree ${ }^{2}$ central part around $L_{4}$. The case $1-\mu=0$ is shown for comparison.

\begin{tabular}{|c|c|c|c|c|c|c|c|c|}
\hline $1-\mu$ & .1 & .01 & $5 \times 10^{-3}$ & $10^{-3}$ & $5 \times 10^{-4}$ & $10^{-4}$ & $10^{-5}$ & 0 \\
\hline $\mathrm{r}[\mathrm{mm}](0=3.5)$ & $1.5 \times 10^{-3}$ & $1.1 \times 10^{-2}$ & $1.9 \times 10^{-2}$ & $7.2 \times 10^{-2}$ & .13 & .49 & 3.3 & - \\
$\mathrm{m}[\mathrm{g}](0=3.5)$ & $6 \times 10^{-11}$ & $2 \times 10^{-8}$ & $10^{-7}$ & $6 \times 10^{-6}$ & $3.4 \times 10^{-5}$ & $1.8 \times 10^{-3}$ & $5.6 \times 10^{-1}$ & - \\
$\mathrm{T}_{\text {tot }[\mathrm{d}]}$ & 65 & 1091 & 2985 & 2066 & 5663 & 6303 & 6642 & 6448 \\
$\mathrm{~T}_{1}[\mathrm{~d}]$ & 15 & 113 & 246 & 749 & 3696 & 5362 & 5747 & 5767 \\
$\mathrm{~T}_{2}[\mathrm{~d}]$ & 0.4 & 1.4 & 1.9 & 7.0 & 24.7 & 48.8 & 52.5 & 54.6 \\
\hline
\end{tabular}

In Table 1 we list so-called lifetimes of the particles in the libration region. For the different values of $1-\mu$ the corresponding particle radii and masses are given for $\rho=3.5 \mathrm{~g} \mathrm{~cm}^{-3}$. T tot is the total lifetime of the particle until it leaves the system or hits the Earth or the Moon. $\mathrm{T}_{1}$ gives the time, the particle spends within an area of $50 \times 26$ degrees ${ }^{2}$ centered at $\mathrm{L}_{4}$, having distances from the Earth between 0.8 and 1.2 (unit of length = mean distance Earth-Moon). The times of residence in a 1 degree ${ }^{2}$ central region around $\mathrm{L}_{4}$ are called $\mathrm{T}_{2}$. They are used to compute the surface brightness.

\section{Origin of the particles}

We computed the origin of the particles using only 227 exactly known trajectories. The major part (62 per cent) of the particles had interplanetary origin, 30 per cent came from the Moon, and 8 per cent of the trajectories started at the Earth. Of the particles with interplanetary origin we computed the heliocentric orbital elements outside the Earth's sphere of influence. The resulting distribution in the $(a, e)-p l a n e$ is shown in Figure 1. 


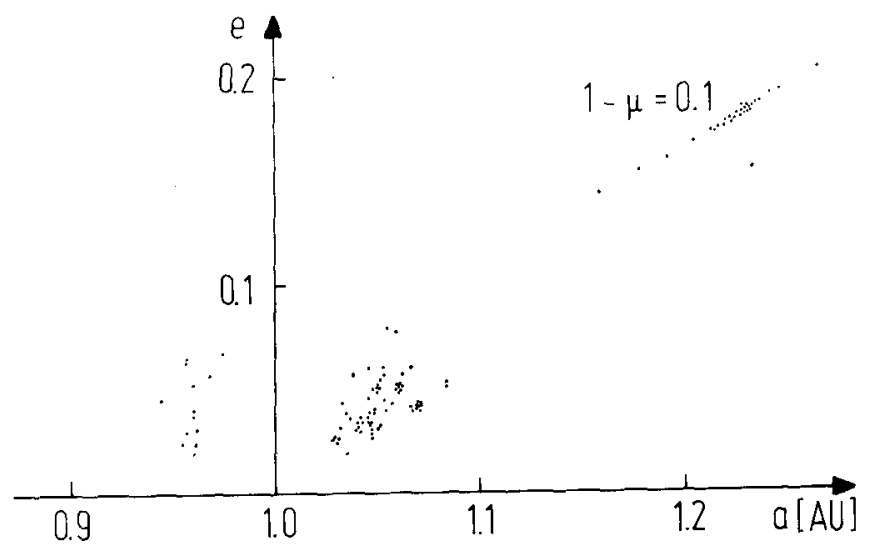

Figure 1. Original values of a (semi-major axis)

and e (eccentricity) for the libration particles

It is remarkable, that the majority of the particles have semi-major axes greater than unity; this can be explained by the action of the Poynting-Robertson drag, which tends to diminish angular momentum and total energy. Of the particles having a $<1$ all exept one had $1-\mu$ values less or equal $10^{-4}$; these are not very much influenced by radiation pressure, as is confirmed by their lifetimes. The remaining particle suffered a near passage by the Moon, which led to a drastic change in its orbital elements.

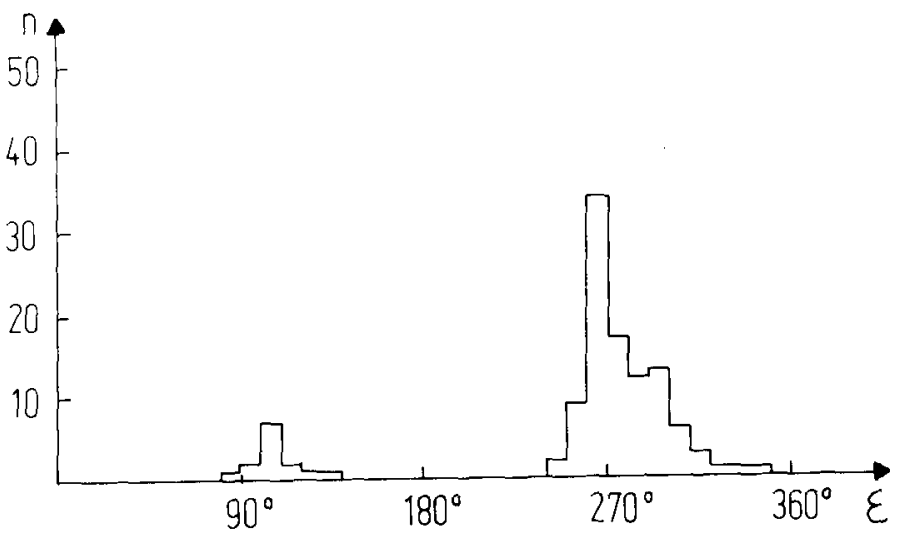

Figure 2. Directions of origin of the libration particles $(\varepsilon=$ Elongation from the Sun ) 
The histogram in Figure 2 gives the distribution of the directions at the sphere, from which the particles must originate, if they are to reach the libration point $\mathrm{I}_{4}$. The abscissa shows elongation from the $\mathrm{Sun}$ and $\mathrm{n}$ is the number of particles coming from a 10 degrees intervall of elongation. The two clearly distinct peaks at the apex and antapex directions of the Earth's orbit are in exact correlation with the results on the orbital elements. In the neighbourhood of the Earth's orbit particles having semi-major axes greater than unity must have velocities which are greater than that of the Earth. They must come from the antapex direction, whereas the contrary is true for the lower energetic particles. The velocities relative to the Earth were very low in all cases. For the smallest particles we found mean relative velocities of about $1.8 \mathrm{~km} \mathrm{sec}{ }^{-1}$, for all others the velocities ranged between 0.8 and 0.9 $\mathrm{km} \sec ^{-1}$.

\section{The surface brightness of the libration cloud}

For the supply of particles to the libration point region we discuss two possible sources, the interplanetary dust particles and the lunar ejecta. We have used recent flux measurements to compute the surface brightness near $\mathrm{L}_{4}$, which is proportional to

i) the lifetime of the particles near $\mathrm{L}_{4}$ as given in Table 1 .

ii) the brightness of a single particle. Instead of a complicated scattering function we assumed totally reflecting particles, which gives an upper limit for the brightness of the cloud.

iii) the amount of mass supplied by the different sources.

For the interplanetary particles we adopted the flux measurements given by Fechtig et al. (1974). The directions at the sphere, from which the particles must originate, are according to Figure 2. To avoid incompleteness we allowed an area of origin of $500 \square^{\circ}$ around the antapex and of $200 \square^{\circ}$ around the apex direction. We made no restriction with respect to the velocity vector, so that the brightness is overestimated considerably. Under these assumptions a surface brightness of $2 \mathrm{~S}_{10 \mathrm{~V}}$ at $\mathrm{L}_{4}$ has been found, a value which can only be understood as a very high upper bound.

The situation is even worse, if we want to discuss the mass supply by the Moon. At present time it is not known, if the Moon gains or looses mass. Nevertheless a rough estimation can be made. From the statements i) and ii) given above, we can compute the mass supply necessary to maintain a surface brightness of $1 s_{10}$ within the central area of $1 \square^{\circ}$ 
around $\mathrm{I}_{4}$. A simple calculation yields

$$
M\left[\mathrm{~kg} \mathrm{~d} \mathrm{~d}^{-1}\right]=7.5 \times\left[\mathrm{T}_{2} \times(1-\mu)\right]^{-1}
$$

The masses needed to compensate the loss amount to 0.8 tons per day for the smallest particles $\left(-10^{-8} \mathrm{~g}\right)$ that can come from the Moon, up to 12.5 tons per day for the largest $\left(\sim 10^{-1} \mathrm{~g}\right)$. According to Hughes (1973) the present influx rate of interplanetary dust to the Earth is $5.7 \times 10^{9} \mathrm{~g} \mathrm{yr}^{-1}$. Applied to the Moon, this means an influx rate of 4 tons per day. Assuming that the Moon looses mass at about the same amount, which is not established by measurements, only between 10 and 20 per cent of it will remain within the Earth-Moon system (Shapiro et al., 1966). Again we did not make any rectriction with respect to velocities, which would diminish the mass supply. So it seems evident that the Moon is out of question as a source.

\section{Conclusion}

Calculations as presented here will always be at a state of incompleteness. The great variety of parameters in the phase space prevents a comprehensive treatment of the problem. Besides these theoretical difficulties the experimental data are not yet at a satisfactory level. Nevertheless even the rough estimations in the last chapter indicate, that the conditions are not favourable for libration clouds, so that we are in doubt if they really exist.

\section{$\underline{\text { References }}$}

Burnett, G.B.; Sparrow, J.G. and Ney, E.P.; Is the Zodiacal Light intensity steady? Nature 249, 639 (1974).

Bruman, J.R.; A Lunar Libration Point Experiment. Icarus 10, 197 (1969).

Fechtig, H.; Gentner, W.; Hartung, J.B.; Nagel, K.; Neukum, G.; Schneider E.; and Storzer, D.; Microcraters on lunar samples. Soviet-American Conference on Cosmochemistry of the moon and the planets, Moscow, 1974.

Hughes, D.W.; Interplanetary Dust and its influx to the Earth's Surface, XVI Plenary Meeting of Cospar, Konstanz, 1973.

Matas, V.; Periodic Solutions of a Disturbed Elliptic Restricted Three - Body - Problem. BACz, 25, 129 (1974).

Roach, J.R.; Counterglow from the Earth - Moon Libration Points, Planetary and Space Science, 23, 173 (1975).

Robertson, H.P.; Dynamical effects of radiation in the solar system MNRAS, 97, 423 (1937).

Shapiro, I.I.; Lautman, D.A.; and Colombo, G.; The Earth's dust belt: Fact or fiction? JGR 71, 5695 (1966). 